\title{
Supplementary Information of
}

\section{A self-sustained system spanning the primary and secondary metabolism stages to boost the productivity of Streptomyces}

Ming Zhao ${ }^{1}$, Mingrui Wang ${ }^{1}$, Shuiling Wang ${ }^{1}$, Liangbin Xiong ${ }^{1,2}$, Bei Gao ${ }^{1}$, Min Liu $^{1}$, Xinyi Tao ${ }^{1 *}$, Feng-Qing Wang ${ }^{1 *}$, Dongzhi Wei ${ }^{1}$

${ }^{1}$ State Key Laboratory of Bioreactor Engineering, Newworld Institute of Biotechnology, East China University of Science and Technology, 130 Meilong Road, Shanghai 200237, China

${ }^{2}$ Shanghai Key Laboratory of Molecular Imaging, Shanghai University of Medicine and Health Sciences, Shanghai 201318, China.

* Corresponding author:

Feng-Qing Wang-State Key Laboratory of Bioreactor Engineering, Newworld Institute of Biotechnology, East China University of Science and Technology, 130 Meilong Road, Shanghai 200237, China; orcid.org/0000-0002-3473-5991; Email: fqwang@ecust.edu.cn

Xinyi Tao-State Key Laboratory of Bioreactor Engineering, Newworld Institute of Biotechnology, East China University of Science and Technology, 130 Meilong Road, Shanghai 200237, China; Email: xytao@ecust.edu.cn 


\section{Contents of supplementary material:}

Table S1. Plasmids used in this study.

Table S2. The sequence of evolutionary promoters' mutation region.

Figure S1. Relative strength of nine $\mathrm{P} k a s O_{\mathrm{R} 15}$ mutants without the regulation of $\mathrm{HrdB}$ in E. coli.

Figure S2. Indigoidine production curves of S. lividans TK24 and S. albus J1074 under the regulation of $\mathrm{PM}$.

Figure S3. UDP production curves of $S$. lividans TK24 under the regulation of PM.

Figure S4. sqRT-PCR of $h r d B$ and indC under the regulation of $\mathrm{PM}_{\mathrm{P} 12-16}$ and $S t \mathrm{SS} 1$ in

\section{S. lividans TK24.}

Figure S5. Functional enrichment analysis of transcripts based on KEGG pathways.

Figure S6. The toxicity of different antibiotics on different Streptomyces species. 
Table S1. Plasmids used in this study.

\begin{tabular}{|c|c|}
\hline Primers & Sequences (5'-3') \\
\hline \multicolumn{2}{|c|}{ Primers for construction of vectors used for the evolution of $\mathrm{PkasO}{ }_{\mathrm{R} 15}$ in $\mathrm{E}$. coli } \\
\hline HrdB-F & AGCAAATGGGTCGCGGATCCATGTCGGCCAGCACATCCCG \\
\hline HrdB-R & TGTCGACGGAGCTCGAATTCCTAGTCGAGGTAGTCGCGC \\
\hline \multirow[t]{3}{*}{$\mathrm{P} k a s{ }^{*}{ }_{\mathrm{R} 15}-\mathrm{GFP}-\mathrm{F}$} & GTATTAATTTCGCGGGATCGTGTTCACATTCGAACCGTCTCTGCTTT \\
\hline & GACAACATGCTGTGCGGTGTTGTAAAGTCGTGGCCAGGAGAATACG \\
\hline & ACAGTCTAAGTAAGGAGTGTCCATATGAGTAAAGGAGAAGAAC \\
\hline \multirow[t]{3}{*}{ SRL-1-GFP-F } & GTATTAATTTCGCGGGATCGTGTTCACATTCGAACCGTCTCTGCTTT \\
\hline & GACAACATGCTGTGCGGTGTTGTAAAGTNNNNNNNAGGAGAATAC \\
\hline & GACAGTCTAAGTAAGGAGTGTCCATATGAGTAAAGGAGAAGAAC \\
\hline \multirow[t]{3}{*}{ SRL-2-GFP-F } & GTATTAATTTCGCGGGATCGTGTTCACATTCGAACCGTCTCTGCTTT \\
\hline & GACANNNNNNNNNNNNNNNNNNTAAAGTCGTGGCCAGGAGAATA \\
\hline & CGACAGTCTAAGTAAGGAGTGTCCATATGAGTAAAGGAGAAGAAC \\
\hline \multirow[t]{3}{*}{$\mathrm{P}_{10-7}-\mathrm{GFP}-\mathrm{F}$} & GTATTAATTTCGCGGGATCGTGTTCACATTCGAACCGTCTCTGCTTT \\
\hline & GACAATTAAGAACATATAAAGGTAAAGTCGTGTCCAGGAGAATAC \\
\hline & GACAGTCTAAGTAAGGAGTGTCCATATGAGTAAAGGAGAAGAAC \\
\hline \multirow[t]{3}{*}{$\mathrm{P}_{12-7}-\mathrm{GFP}-\mathrm{F}$} & GTATTAATTTCGCGGGATCGTGTTCACATTCGAACCGTCTCTGCTTT \\
\hline & GACAATTAAGAACATATAAAGGTAAAGTAGAACACAGGAGAATAC \\
\hline & GACAGTCTAAGTAAGGAGTGTCCATATGAGTAAAGGAGAAGAAC \\
\hline \multirow[t]{3}{*}{$\mathrm{P}_{16-7-\mathrm{GFP}-\mathrm{F}}$} & GTATTAATTTCGCGGGATCGTGTTCACATTCGAACCGTCTCTGCTTT \\
\hline & GACAATTAAGAACATATAAAGGTAAAGTCTCGACCAGGAGAATAC \\
\hline & GACAGTCTAAGTAAGGAGTGTCCATATGAGTAAAGGAGAAGAAC \\
\hline \multirow[t]{3}{*}{$\mathrm{P}_{10-16^{-}}$GFP-F } & GTATTAATTTCGCGGGATCGTGTTCACATTCGAACCGTCTCTGCTTT \\
\hline & GACAAGCCCCTCTTTCGTACTGTAAAGTCGTGTCCAGGAGAATACG \\
\hline & ACAGTCTAAGTAAGGAGTGTCCATATGAGTAAAGGAGAAGAAC \\
\hline \multirow[t]{3}{*}{$\mathrm{P}_{12-1 \sigma^{-}} \mathrm{GFP}-\mathrm{F}$} & GTATTAATTTCGCGGGATCGTGTTCACATTCGAACCGTCTCTGCTTT \\
\hline & GACAAGCCCCTCTTTCGTACTGTAAAGTAGAACACAGGAGAATACG \\
\hline & ACAGTCTAAGTAAGGAGTGTCCATATGAGTAAAGGAGAAGAAC \\
\hline \multirow[t]{3}{*}{$\mathrm{P}_{16-16^{-} \mathrm{GFP}-\mathrm{F}}$} & GTATTAATTTCGCGGGATCGTGTTCACATTCGAACCGTCTCTGCTTT \\
\hline & GACAAGCCCCTCTTTCGTACTGTAAAGTCTCGACCAGGAGAATACG \\
\hline & ACAGTCTAAGTAAGGAGTGTCCATATGAGTAAAGGAGAAGAAC \\
\hline GFP-R & GCGTCCGGCGTAGAGGATCGTTATTTGTATAGTTCATCC \\
\hline \multicolumn{2}{|c|}{ Primers for construction of vectors used for the evaluation of $\mathrm{PkasO}{ }^{*}{ }_{\mathrm{R} 15}$ mutants in $\mathrm{S}$. lividans } \\
\hline \multirow[t]{3}{*}{$\mathrm{Pkas} O_{\mathrm{R} 15}$-GusA-F } & ACGTCCGCGAGCTGGGATCCTGTTCACATTCGAACCGTCTCTGCTTT \\
\hline & GACAACATGCTGTGCGGTGTTGTAAAGTCGTGGCCAGGAGAATACG \\
\hline & ACAGTCTAAGTAAGGAGTGTCCATATGTTACGTCCTGTAGAAAC \\
\hline \multirow[t]{3}{*}{$\mathrm{P}_{1-10}$-GusA-F } & ACGTCCGCGAGCTGGGATCCTGTTCACATTCGAACCGTCTCTGCTTT \\
\hline & GACAACATGCTGTGCGGTGTTGTAAAGTCGTGTCCAGGAGAATACG \\
\hline & ACAGTCTAAGTAAGGAGTGTCCATATGTTACGTCCTGTAGAAAC \\
\hline \multirow[t]{2}{*}{$\mathrm{P}_{1-12}$-GusA-F } & $\begin{array}{l}\text { ACGTCCGCGAGCTGGGATCCTGTTCACATTCGAACCGTCTCTGCTTT } \\
\end{array}$ \\
\hline & GACAACATGCTGTGCGGTGTTGTAAAGTAGAACACAGGAGAATAC \\
\hline
\end{tabular}




\begin{tabular}{|c|c|}
\hline & GACAGTCTAAGTAAGGAGTGTCCATATGTTACGTCCTGTAGAAAC \\
\hline \multirow[t]{3}{*}{$\mathrm{P}_{1-14}$-GusA-F } & ACGTCCGCGAGCTGGGATCCTGTTCACATTCGAACCGTCTCTGCTTT \\
\hline & GACAACATGCTGTGCGGTGTTGTAAAGTGCACCCGAGGAGAATAC \\
\hline & GACAGTCTAAGTAAGGAGTGTCCATATGTTACGTCCTGTAGAAAC \\
\hline \multirow[t]{3}{*}{$\mathrm{P}_{1-15}$-GusA-F } & ACGTCCGCGAGCTGGGATCCTGTTCACATTCGAACCGTCTCTGCTTT \\
\hline & GACAACATGCTGTGCGGTGTTGTAAAGTAGCACAGAGGAGAATAC \\
\hline & GACAGTCTAAGTAAGGAGTGTCCATATGTTACGTCCTGTAGAAAC \\
\hline \multirow[t]{3}{*}{$\mathrm{P}_{1-16^{-} \mathrm{GusA} A-\mathrm{F}}$} & ACGTCCGCGAGCTGGGATCCTGTTCACATTCGAACCGTCTCTGCTTT \\
\hline & GACAACATGCTGTGCGGTGTTGTAAAGTCTCGACCAGGAGAATACG \\
\hline & ACAGTCTAAGTAAGGAGTGTCCATATGTTACGTCCTGTAGAAAC \\
\hline \multirow{3}{*}{$\mathrm{P}_{2-4^{-}}$GusA-F } & ACGTCCGCGAGCTGGGATCCTGTTCACATTCGAACCGTCTCTGCTTT \\
\hline & GACAATATGCCCAACGCAAAGGTAAAGTCGTGGCCAGGAGAATAC \\
\hline & GACAGTCTAAGTAAGGAGTGTCCATATGTTACGTCCTGTAGAAAC \\
\hline \multirow[t]{3}{*}{$\mathrm{P}_{2-5^{-}}$GusA-F } & ACGTCCGCGAGCTGGGATCCTGTTCACATTCGAACCGTCTCTGCTTT \\
\hline & GACAAGCCCCTCTTTCGTACTCTAAAGTCGTGGCCAGGAGAATACG \\
\hline & ACAGTCTAAGTAAGGAGTGTCCATATGTTACGTCCTGTAGAAAC \\
\hline \multirow[t]{3}{*}{$\mathrm{P}_{2-6^{-} \text {-GusA-F }}$} & $\begin{array}{l}\text { ACGTCCGCGAGCTGGGATCCTGTTCACATTCGAACCGTCTCTGCTTT } \\
\end{array}$ \\
\hline & GACAACACGCTTCGTTCATCTCTAAAGTCGTGGCCAGGAGAATACG \\
\hline & ACAGTCTAAGTAAGGAGTGTCCATATGTTACGTCCTGTAGAAAC \\
\hline \multirow{3}{*}{$\mathrm{P}_{2-7}$-GusA-F } & ACGTCCGCGAGCTGGGATCCTGTTCACATTCGAACCGTCTCTGCTTT \\
\hline & GACAATTAAGAACATATAAAGGTAAAGTCGTGGCCAGGAGAATAC \\
\hline & GACAGTCTAAGTAAGGAGTGTCCATATGTTACGTCCTGTAGAAAC \\
\hline \multirow[t]{3}{*}{$\mathrm{P}_{2-16}-\mathrm{GusA}-\mathrm{F}$} & ACGTCCGCGAGCTGGGATCCTGTTCACATTCGAACCGTCTCTGCTTT \\
\hline & GACAAGCCCCTCTTTCGTACTGTAAAGTCGTGGCCAGGAGAATACG \\
\hline & ACAGTCTAAGTAAGGAGTGTCCATATGTTACGTCCTGTAGAAAC \\
\hline \multirow[t]{3}{*}{$\mathrm{P}_{10-7}-\mathrm{GusA}-\mathrm{F}$} & ACGTCCGCGAGCTGGGATCCTGTTCACATTCGAACCGTCTCTGCTTT \\
\hline & GACAATTAAGAACATATAAAGGTAAAGTCGTGTCCAGGAGAATAC \\
\hline & GACAGTCTAAGTAAGGAGTGTCCATATGTTACGTCCTGTAGAAAC \\
\hline \multirow[t]{3}{*}{$\mathrm{P}_{12-7}$-GusA-F } & ACGTCCGCGAGCTGGGATCCTGTTCACATTCGAACCGTCTCTGCTTT \\
\hline & GACAATTAAGAACATATAAAGGTAAAGTAGAACACAGGAGAATAC \\
\hline & GACAGTCTAAGTAAGGAGTGTCCATATGTTACGTCCTGTAGAAAC \\
\hline \multirow[t]{3}{*}{$\mathrm{P}_{16-7}-\mathrm{GusA}-\mathrm{F}$} & ACGTCCGCGAGCTGGGATCCTGTTCACATTCGAACCGTCTCTGCTTT \\
\hline & GACAATTAAGAACATATAAAGGTAAAGTCTCGACCAGGAGAATAC \\
\hline & GACAGTCTAAGTAAGGAGTGTCCATATGTTACGTCCTGTAGAAAC \\
\hline \multirow[t]{3}{*}{$\mathrm{P}_{10-16}-\mathrm{GusA-F}$} & ACGTCCGCGAGCTGGGATCCTGTTCACATTCGAACCGTCTCTGCTTT \\
\hline & GACAAGCCCCTCTTTCGTACTGTAAAGTCGTGTCCAGGAGAATACG \\
\hline & ACAGTCTAAGTAAGGAGTGTCCATATGTTACGTCCTGTAGAAAC \\
\hline \multirow[t]{3}{*}{$\mathrm{P}_{12-16}-\mathrm{GusA}-\mathrm{F}$} & ACGTCCGCGAGCTGGGATCCTGTTCACATTCGAACCGTCTCTGCTTT \\
\hline & GACAAGCCCCTCTTTCGTACTGTAAAGTAGAACACAGGAGAATACG \\
\hline & ACAGTCTAAGTAAGGAGTGTCCATATGTTACGTCCTGTAGAAAC \\
\hline \multirow[t]{3}{*}{$\mathrm{P}_{16-16}$-GusA-F } & ACGTCCGCGAGCTGGGATCCTGTTCACATTCGAACCGTCTCTGCTTT \\
\hline & GACAAGCCCCTCTTTCGTACTGTAAAGTCTCGACCAGGAGAATACG \\
\hline & ACAGTCTAAGTAAGGAGTGTCCATATGTTACGTCCTGTAGAAAC \\
\hline
\end{tabular}




\begin{tabular}{|c|c|}
\hline GusA-R & GGGAACGCGTAGATCTGAATTCTCATTGTTTGCCTCCCTGC \\
\hline pFQ-F & GAGAATTCAGATCTACGCGTTCCCCGC \\
\hline pFQ-R & CCGGATCCCAGCTCGCGGACGTGCTCATAG \\
\hline \multicolumn{2}{|c|}{ Primers for construction of vectors used for the expression of indC gene in Streptomyces } \\
\hline \multirow[t]{3}{*}{$\mathrm{P} k a s{ }^{*}{ }_{\mathrm{R} 15}$-IndC-F } & ACGTCCGCGAGCTGGGATCCTGTTCACATTCGAACCGTCTCTGCTTT \\
\hline & GACAACATGCTGTGCGGTGTTGTAAAGTCGTGGCCAGGAGAATACG \\
\hline & ACAGTCTAAGTAAGGAGTGTCCATATGCCGTCTCGCACTCCC \\
\hline \multirow[t]{3}{*}{$\mathrm{P}_{1-12 \text {-IndC-F }}$} & ACGTCCGCGAGCTGGGATCCTGTTCACATTCGAACCGTCTCTGCTTT \\
\hline & GACAACATGCTGTGCGGTGTTGTAAAGTAGAACACAGGAGAATAC \\
\hline & GACAGTCTAAGTAAGGAGTGTCCATATGCCGTCTCGCACTCCC \\
\hline \multirow[t]{3}{*}{$\mathrm{P}_{1-16}$-IndC-F } & ACGTCCGCGAGCTGGGATCCTGTTCACATTCGAACCGTCTCTGCTTT \\
\hline & GACAACATGCTGTGCGGTGTTGTAAAGTCTCGACCAGGAGAATACG \\
\hline & ACAGTCTAAGTAAGGAGTGTCCATATGCCGTCTCGCACTCCC \\
\hline \multirow[t]{3}{*}{$\mathrm{P}_{2-7}-$ IndC-F } & ACGTCCGCGAGCTGGGATCCTGTTCACATTCGAACCGTCTCTGCTTT \\
\hline & GACAATTAAGAACATATAAAGGTAAAGTCGTGGCCAGGAGAATAC \\
\hline & GACAGTCTAAGTAAGGAGTGTCCATATGCCGTCTCGCACTCCC \\
\hline \multirow[t]{3}{*}{$\mathrm{P}_{2-16}$-IndC-F } & ACGTCCGCGAGCTGGGATCCTGTTCACATTCGAACCGTCTCTGCTTT \\
\hline & GACAAGCCCCTCTTTCGTACTGTAAAGTCGTGGCCAGGAGAATACG \\
\hline & ACAGTCTAAGTAAGGAGTGTCCATATGCCGTCTCGCACTCCC \\
\hline \multirow[t]{3}{*}{$\mathrm{P}_{10-16}$-IndC-F } & ACGTCCGCGAGCTGGGATCCTGTTCACATTCGAACCGTCTCTGCTTT \\
\hline & GACAAGCCCCTCTTTCGTACTGTAAAGTCGTGTCCAGGAGAATACG \\
\hline & ACAGTCTAAGTAAGGAGTGTCCATATGCCGTCTCGCACTCCC \\
\hline \multirow[t]{3}{*}{$\mathrm{P}_{12-16}-$ IndC-F } & ACGTCCGCGAGCTGGGATCCTGTTCACATTCGAACCGTCTCTGCTTT \\
\hline & GACAAGCCCCTCTTTCGTACTGTAAAGTAGAACACAGGAGAATACG \\
\hline & ACAGTCTAAGTAAGGAGTGTCCATATGCCGTCTCGCACTCCC \\
\hline $\mathrm{P}_{R V R 2353}-\mathrm{F}$ & ACGTCCGCGAGCTGGGATCCCACCTGGACATCGCGG \\
\hline $\mathrm{P}_{R V R 2353}-\mathrm{R}$ & GCGGGAGTGCGAGACGGCATGTCACGTCCTCCATGC \\
\hline $\mathrm{P}_{R V R 3650}-\mathrm{F}$ & ACGTCCGCGAGCTGGGATCCACCAGCATCCCCAAGGG \\
\hline $\mathrm{P}_{R V R 3650}-\mathrm{R}$ & GCGGGAGTGCGAGACGGCATGTTGTCTCCTTCTGAGATG \\
\hline $\mathrm{P}_{R V R 6682} \mathrm{~F}$ & ACGTCCGCGAGCTGGGATCCTGCCCAACATCTGGAGC \\
\hline $\mathrm{P}_{R V R 6682}-\mathrm{R}$ & GCGGGAGTGCGAGACGGCATGAAATCCTGCCTGTCTGG \\
\hline $\mathrm{P}_{R V R 7941}-\mathrm{F}$ & ACGTCCGCGAGCTGGGATCCCCTCTGCAACCTGCTCAAC \\
\hline $\mathrm{P}_{R V R 7941}-\mathrm{R}$ & GCGGGAGTGCGAGACGGCATCTGAGGTTGCCTCCCTGAC \\
\hline $\mathrm{P}_{R V R 9107}-\mathrm{F}$ & ACGTCCGCGAGCTGGGATCCCGTACCCATCTGCCGCTC \\
\hline $\mathrm{P}_{\text {RVR9107 }}-\mathrm{R}$ & GCGGGAGTGCGAGACGGCATGGCGGGGTCCTCCGGAGG \\
\hline IndC-F & ATGCCGTCTCGCACTCCCGCAGGGC \\
\hline IndC-R & GAACGCGTAGATCTGAATTCCTAGGCGAAGAGGTCCAAGG \\
\hline \multicolumn{2}{|c|}{ Primers for construction of vectors used for the expression of EGT BGC in Streptomyces } \\
\hline \multirow[t]{3}{*}{$\mathrm{P}_{12-16}$-EGT-F } & ACGTCCGCGAGCTGGGATCCTGTTCACATTCGAACCGTCTCTGCTTT \\
\hline & GACAAGCCCCTCTTTCGTACTGTAAAGTAGAACACAGGAGAATACG \\
\hline & ACAGTCTAAGTAAGGAGTGTCCATGTGGTTCCGATGTCGGCC \\
\hline EGT-R & GAACGCGTAGATCTGAATTCCTACGAACTGACGGCCATCAACGCCG \\
\hline
\end{tabular}




\begin{tabular}{|c|c|}
\hline $\mathrm{P}_{12-16^{-a v e R}-\mathrm{F}}$ & $\begin{array}{l}\text { ACGTCCGCGAGCTGGGATCCTGTTCACATTCGAACCGTCTCTGCTTT } \\
\text { GACAAGCCCCTCTTTCGTACTGTAAAGTAGAACACAGGAGAATACG } \\
\text { ACAGTCTAAGTAAGGAGTGTCCATGCAGGGAGTTTCCTGTC }\end{array}$ \\
\hline aveR-R & GAACGCGTAGATCTGAATTCTCAGTTGGCCTGGTCCGCG \\
\hline \multicolumn{2}{|c|}{ Primers for construction of vectors used for the expression of $h r d B$ gene in S. lividans } \\
\hline $\mathrm{P}_{R V R 2353}$-HrdB-F & GCGAGTATCTGAAAGGGGATCACCTGGACATCGCGGGC \\
\hline $\mathrm{P}_{R V R 2353}-\mathrm{HrdB}-\mathrm{R}$ & GGGATGTGCTGGCCGACATGTCACGTCCTCCATGCATG \\
\hline $\mathrm{P}_{R V R 6682}-\mathrm{HrdB}-\mathrm{F}$ & GCGAGTATCTGAAAGGGGATTGCCCAACATCTGGAGC \\
\hline $\mathrm{P}_{R V R 6682}-\mathrm{HrdB}-\mathrm{R}$ & CGGGATGTGCTGGCCGACATGAAATCCTGCCTGTCTGG \\
\hline HrdB-F-2 & ATGTCGGCCAGCACATCCCGTACGCTC \\
\hline HrdB-R-2 & GCTATTTCTAGCTCTAAAACCTAGTCGAGGTAGTCGC \\
\hline \multicolumn{2}{|c|}{ Primers for construction of vectors used for knock-in of promoters into $S$. lividans genome } \\
\hline gRNA-red-F & ACGCGCCCGAATCCGATCGTTCGG \\
\hline gRNA-red-R & AAACCCGAACGATCGGATTCGGGC \\
\hline RED-LHA-F & CCGGGCGTTTTTTATCTAGAAGGGCGCCTCGGCGGACCAG \\
\hline RED-LHA-R & GACGGTTCGAATGTGAACACTGCGCCCCCGTCGAGATTC \\
\hline $\mathrm{P} k a s{ }^{*}{ }_{\mathrm{R} 15}-\mathrm{RHA}-\mathrm{F}$ & $\begin{array}{l}\text { TGTTCACATTCGAACCGTCTCTGCTTTGACAACATGCTGTGCGGTGT } \\
\text { TGTAAAGTCGTGGCCAGGAGAATACGACAGTCTAAGTAAGGAGTG } \\
\text { TCCATATGAGATTCAACTTATTG }\end{array}$ \\
\hline $\mathrm{P}_{1-12}$-RHA-F & $\begin{array}{l}\text { TGTTCACATTCGAACCGTCTCTGCTTTGACAACATGCTGTGCGGTGT } \\
\text { TGTAAAGTAGAACACAGGAGAATACGACAGTCTAAGTAAGGAGTG } \\
\text { TCCATATGAGATTCAACTTATTG }\end{array}$ \\
\hline $\mathrm{P}_{1-16}$-RHA-F & $\begin{array}{l}\text { TGTTCACATTCGAACCGTCTCTGCTTTGACAACATGCTGTGCGGTGT } \\
\text { TGTAAAGTCTCGACCAGGAGAATACGACAGTCTAAGTAAGGAGTG } \\
\text { TCCATATGAGATTCAACTTATTG }\end{array}$ \\
\hline $\mathrm{P}_{2-7}$-RHA-F & $\begin{array}{l}\text { TGTTCACATTCGAACCGTCTCTGCTTTGACAATTAAGAACATATAA } \\
\text { AGGTAAAGTCGTGGCCAGGAGAATACGACAGTCTAAGTAAGGAGT } \\
\text { GTCCATATGAGATTCAACTTATTG }\end{array}$ \\
\hline $\mathrm{P}_{2-16}$-RHA-F & $\begin{array}{l}\text { TGTTCACATTCGAACCGTCTCTGCTTTGACAAGCCCCTCTTTCGTAC } \\
\text { TGTAAAGTCGTGGCCAGGAGAATACGACAGTCTAAGTAAGGAGTG } \\
\text { TCCATATGAGATTCAACTTATTG }\end{array}$ \\
\hline $\mathrm{P}_{10-16}$-RHA-F & $\begin{array}{l}\text { TGTTCACATTCGAACCGTCTCTGCTTTGACAAGCCCCTCTTTCGTAC } \\
\text { TGTAAAGTCGTGTCCAGGAGAATACGACAGTCTAAGTAAGGAGTGT } \\
\text { CCATATGAGATTCAACTTATTG }\end{array}$ \\
\hline $\mathrm{P}_{12-16}$-RHA-F & $\begin{array}{l}\text { TGTTCACATTCGAACCGTCTCTGCTTTGACAAGCCCCTCTTTCGTAC } \\
\text { TGTAAAGTAGAACACAGGAGAATACGACAGTCTAAGTAAGGAGTG } \\
\text { TCCATATGAGATTCAACTTATTG }\end{array}$ \\
\hline RED-RHA-R & CCTTTTTACGGTTCCTGGCCACCGAGGAGGCCTTCGACC \\
\hline
\end{tabular}


Table S2. The sequence of evolutionary promoters' mutation region.

\begin{tabular}{|c|c|}
\hline Promoter & Sequence of mutation region (5' ${ }^{\prime}{ }^{\prime}$ ) \\
\hline PkasO ${ }^{*}{ }_{\mathrm{R}} 15$ & TTGACAACATGCTGTGCGGTGTTGTAAAGTCGTGGCCA \\
\hline SRL-1-10 & TTGACAACATGCTGTGCGGTGTTGTAAAGTCGTGTCCA \\
\hline SRL-1-12 & TTGACAACATGCTGTGCGGTGTTGTAAAGTAGAACACA \\
\hline SRL-1-14 & TTGACAACATGCTGTGCGGTGTTGTAAAGTGCACCCGA \\
\hline SRL-1-15 & TTGACAACATGCTGTGCGGTGTTGTAAAGTAGCACAGA \\
\hline SRL-1-16 & TTGACAACATGCTGTGCGGTGTTGTAAAGTCTCGACCA \\
\hline SRL-2-4 & TTGACAATATGCCCAACGCAAAGGTAAAGTCGTGGCCA \\
\hline SRL-2-5 & TTGACAAGCCCCTCTTTCGTACTCTAAAGTCGTGGCCA \\
\hline SRL-2-6 & TTGACAACACGCTTCGTTCATCTCTAAAGTCGTGGCCA \\
\hline SRL-2-7 & TTGACAATTAAGAACATATAAAGGTAAAGTCGTGGCCA \\
\hline SRL-2-16 & TTGACAAGCCCCTCTTTCGTACTGTAAAGTCGTGGCCA \\
\hline SRL-10-7 & TTGACAATTAAGAACATATAAAGGTAAAGTCGTGTCCA \\
\hline SRL-12-7 & TTGACAATTAAGAACATATAAAGGTAAAGTAGAACACA \\
\hline SRL-16-7 & TTGACAATTAAGAACATATAAAGGTAAAGTCTCGACCA \\
\hline SRL-10-16 & TTGACAAGCCCCTCTTTCGTACTGTAAAGTCGTGTCCA \\
\hline SRL-12-16 & TTGACAAGCCCCTCTTTCGTACTGTAAAGTAGAACACA \\
\hline
\end{tabular}

Notes: Red and purple bases represent -35 region and -10 region, respectively.

Transcription start site is indicated by bigger letters. Mutation bases are indicated by bold letters. 


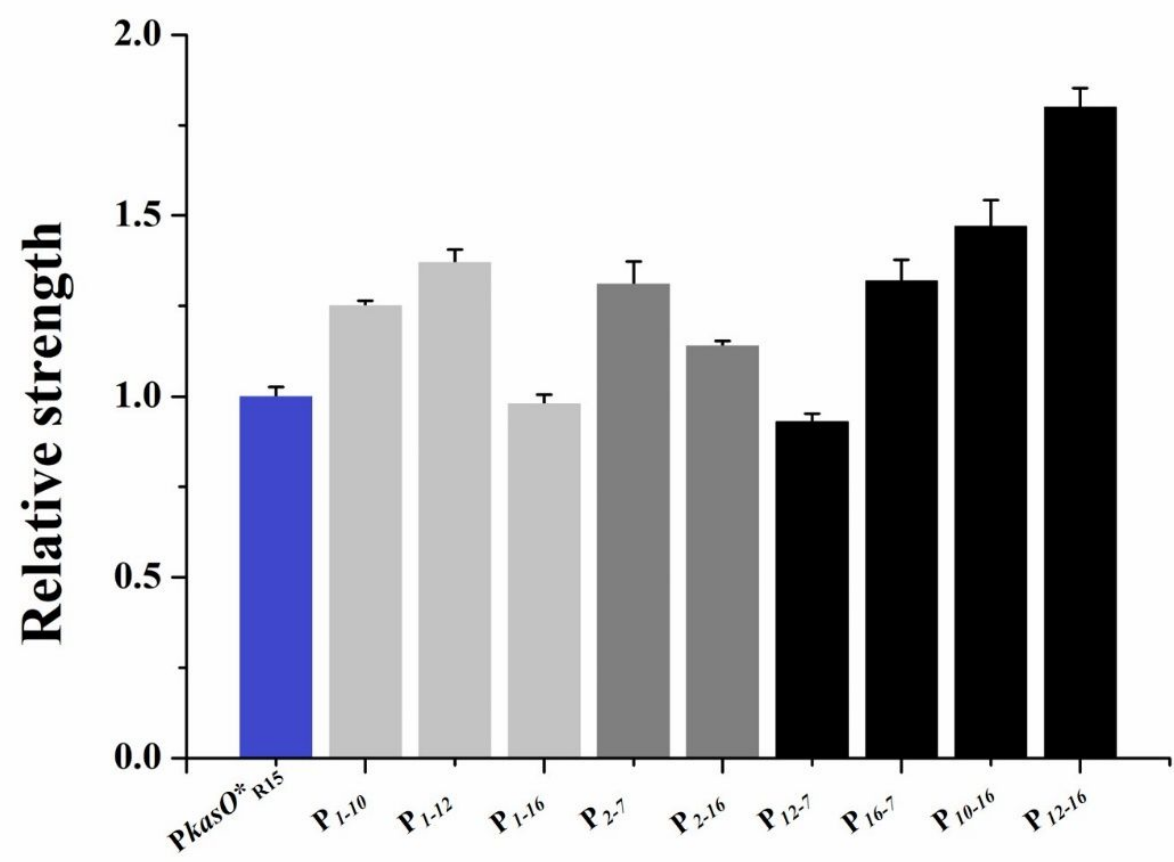

Figure S1. Relative strength of nine $\mathrm{P} k a s O_{\mathrm{R} 15}$ mutants without the regulation of $\mathrm{HrdB}$ in E. coli. Error bars show standard deviation from three independent experiments. 


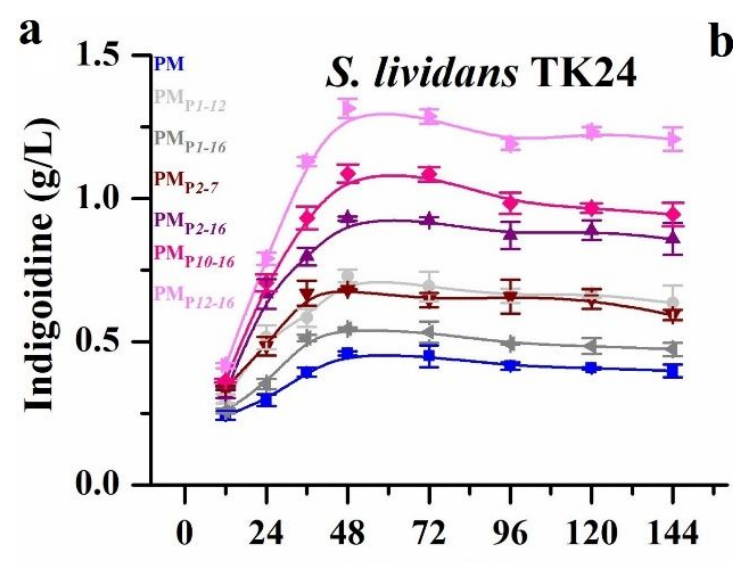

Time (h)

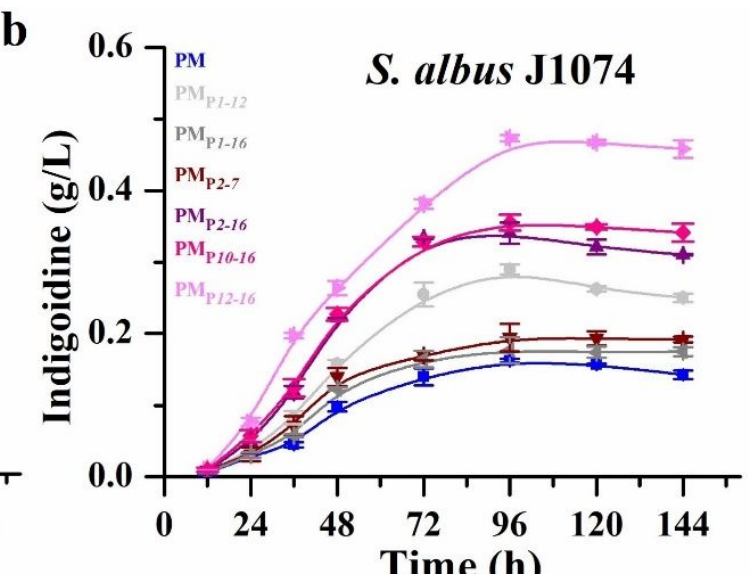

Time (h)

Figure S2. Indigoidine production curves of S. lividans TK24 and S. albus J1074 under the regulation of PM. Error bars show standard deviation from three independent experiments. 


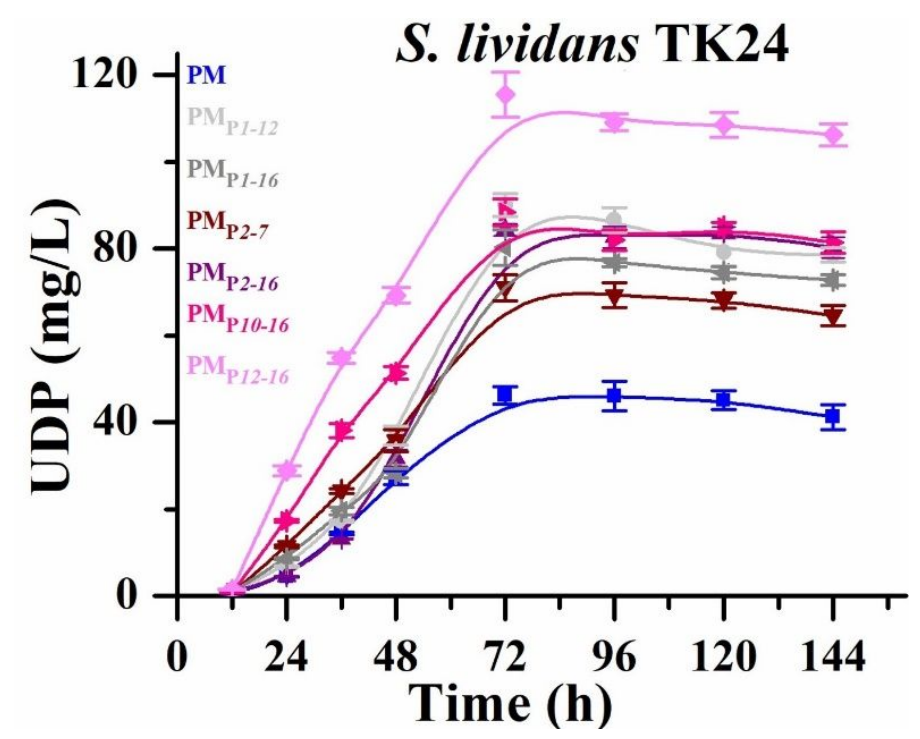

Figure S3. UDP production curves of S. lividans TK24 under the regulation of PM.

Error bars show standard deviation from three independent experiments. 


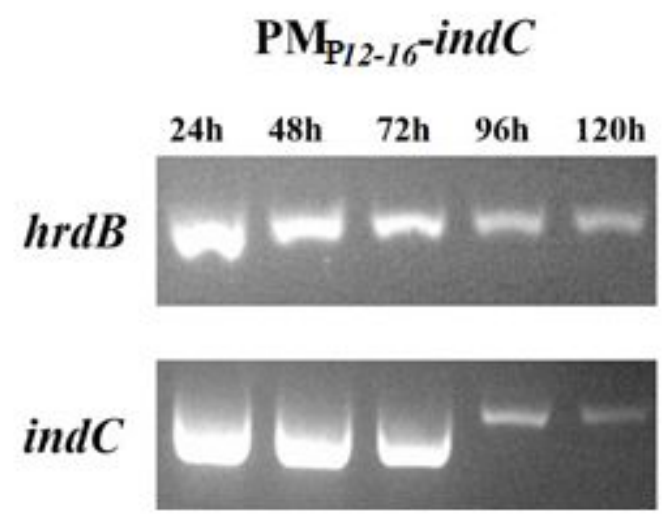

StSS1-indC
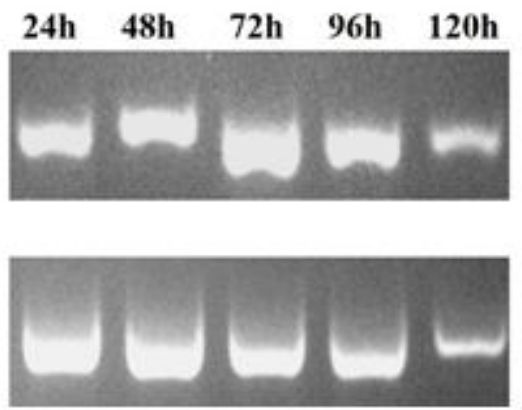

Figure S4. sqRT-PCR of $h r d B$ and $i n d C$ under the regulation of $\mathrm{PM}_{\mathrm{P} 12-16}$ and $S t \mathrm{SS} 1$ in S. lividans TK24. 


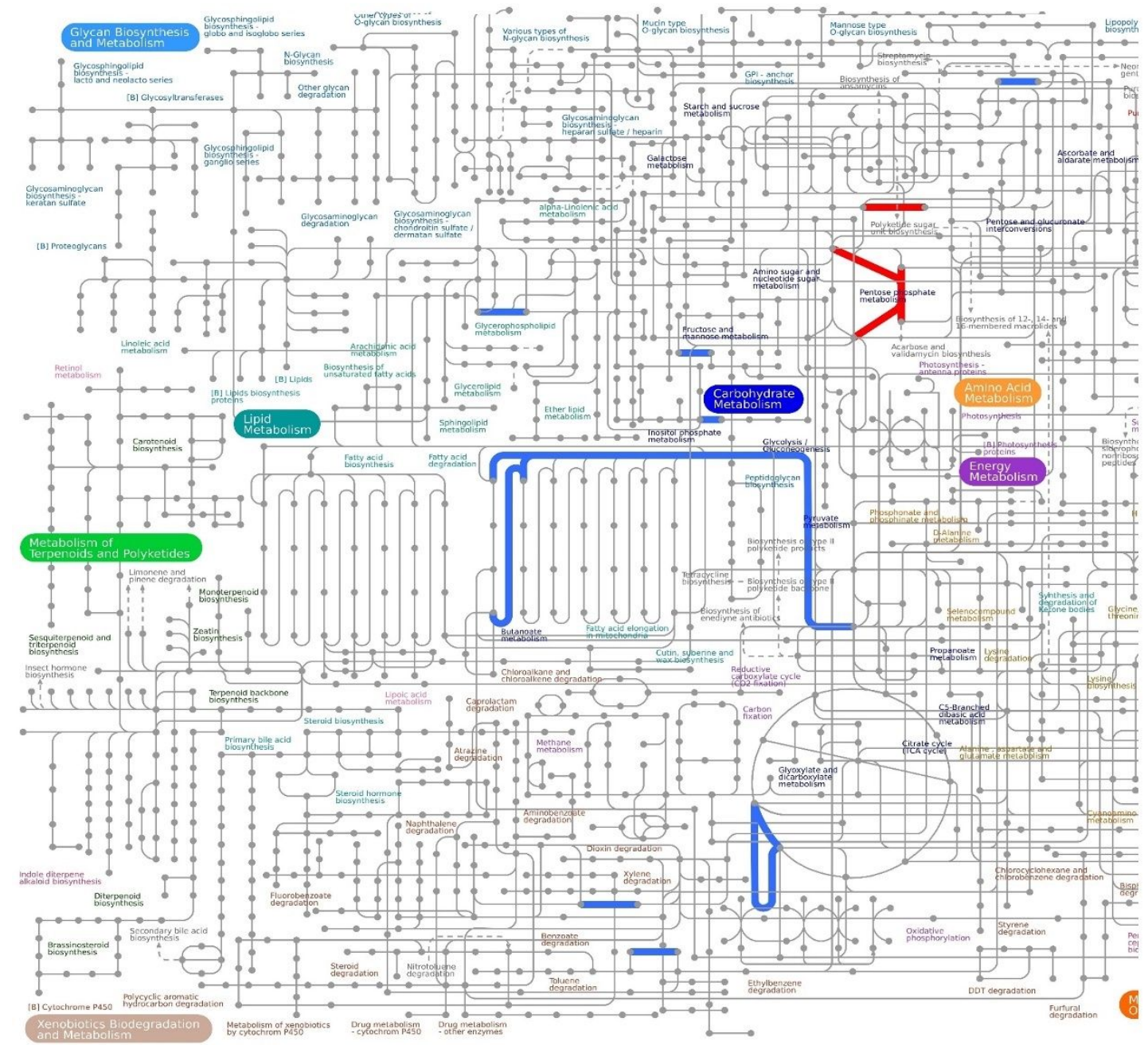

Figure S5. Functional enrichment analysis of transcripts based on KEGG pathways.

Red and blue lines represent the upregulated and downregulated pathways, respectively. 


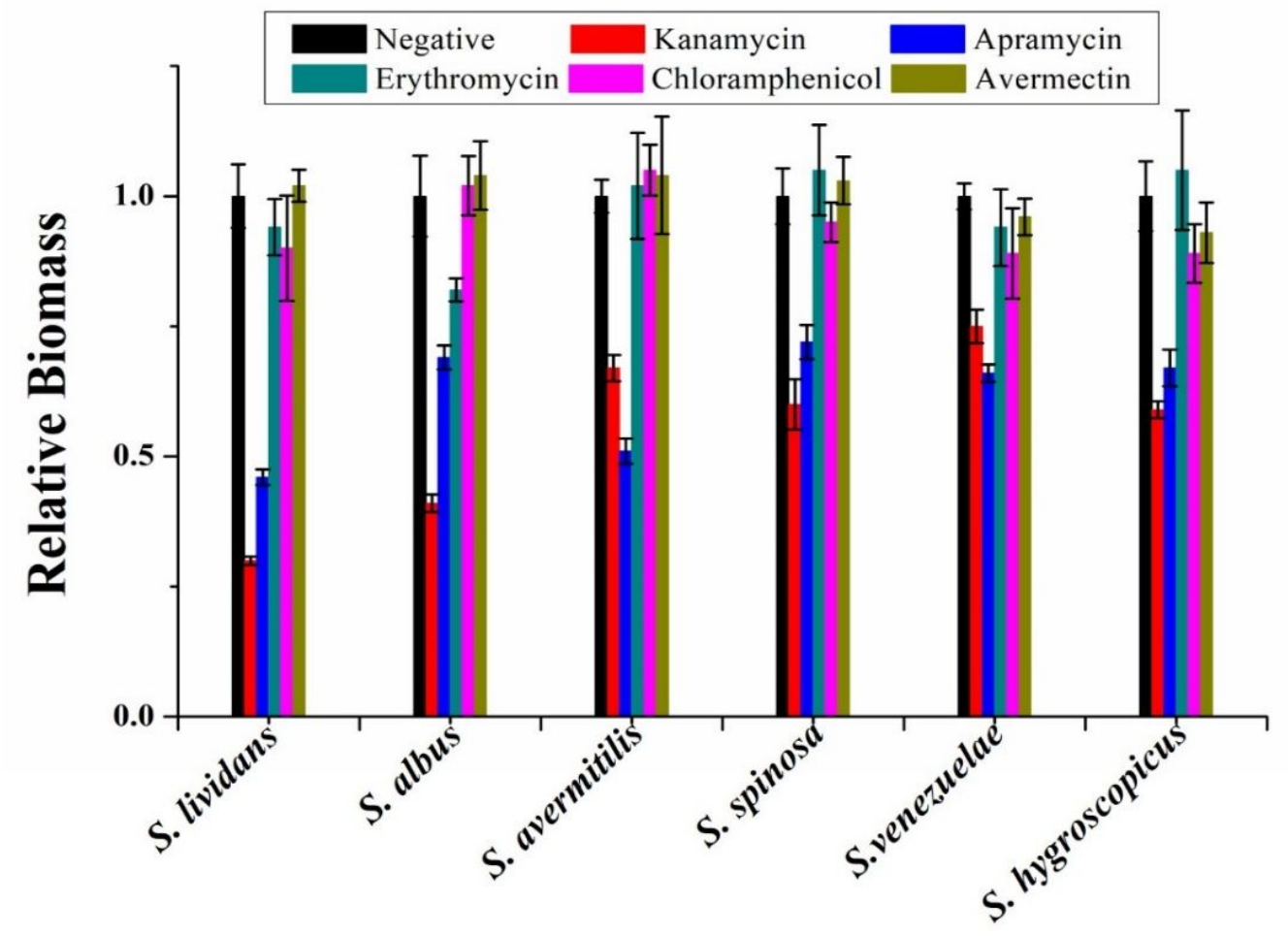

Figure S6. The toxicity of different antibiotics on different Streptomyces species. $1 \mathrm{~g} / \mathrm{L}$ different kinds of antibiotics were added to media, respectively. Relative biomass of each Streptomyces was analyzed after $48 \mathrm{~h}$ of culture. Error bars show standard deviation from three independent experiments. 